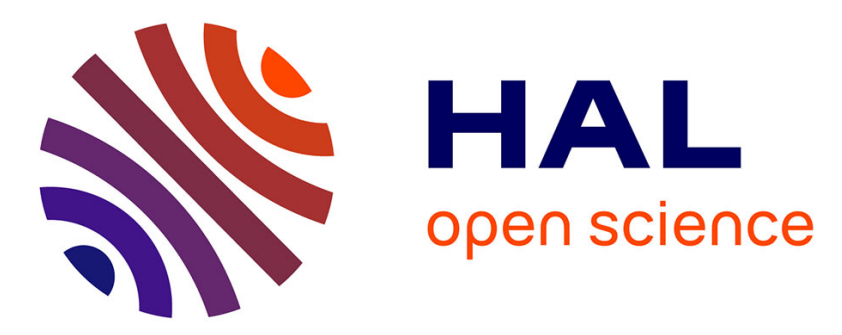

\title{
Time-correlation functions for new cross-multipole field fluctuations in binary light scattering by unlike polar molecules
}

S. Kielich

\section{- To cite this version:}

S. Kielich. Time-correlation functions for new cross-multipole field fluctuations in binary light scattering by unlike polar molecules. Journal de Physique Lettres, 1982, 43 (11), pp.389-394. 10.1051/jphyslet:019820043011038900 . jpa-00232065

\section{HAL Id: jpa-00232065 https://hal.science/jpa-00232065}

Submitted on 1 Jan 1982

HAL is a multi-disciplinary open access archive for the deposit and dissemination of scientific research documents, whether they are published or not. The documents may come from teaching and research institutions in France or abroad, or from public or private research centers.
L'archive ouverte pluridisciplinaire HAL, est destinée au dépôt et à la diffusion de documents scientifiques de niveau recherche, publiés ou non, émanant des établissements d'enseignement et de recherche français ou étrangers, des laboratoires publics ou privés. 
Classification

Physics Abstracts

$33.20 \mathrm{~F}-35.20 \mathrm{M}-78.35$

\title{
Time-correlation functions for new cross-multipole field fluctuations in binary light scattering by unlike polar molecules
}

\author{
S. Kielich \\ Nonlinear Optics Division, Institute of Physics, A. Mickiewicz University, 60-780 Poznań, Poland
}

(Reçu le 24 février 1982, accepté le 14 avril 1982)

\begin{abstract}
Résumé. - Le formalisme des tenseurs multipolaires Cartésiens est appliqué à l'analyse d'un nouveau mécanisme de diffusion de la lumière, dû à l'interférence des variations des polarisabilités moléculaires linéaires et non linéaires induites par les fluctuations temporelles des champs multipolaires électriques. Des formules générales sont proposées pour les fonctions de corrélation temporelles de diffusion isotrope et anisotrope, où entrent les multipolarités de rang arbitraire. Ces formules sont appliquées aux approximations quadrupolaire, octupolaire et hexadécapolaire de molécules à symétrie axiale, tétraédrique et octaédrique.
\end{abstract}

\begin{abstract}
Multipole Cartesian tensor formalism is applied to analyse a new mechanism of light scattering due to the interference of changes in linear and nonlinear molecular polarizabilities induced by space-time fluctuations of multipole electric fields. General formulae are derived for the binary time-correlation functions of isotropic and anisotropic scattering involving multipolarities of arbitrary rank and are applied to the quadrupole, octupole and hexadecapole approximations of simple axially, tetrahedrally and octahedrally symmetric molecules.
\end{abstract}

1. Introduction. - Previously [1], we developed the theory of linear and nonlinear multipole polarizabilities for unlike interacting molecules and applied it to the analysis of deviations from additivity of the Clausius-Mosotti and Lorentz-Lorenz function of dense multi-component fluids. In the present Communication we proceed to apply that consistent multipole Cartesian tensor formalism in order to disclose a novel cross-fluctuation mechanism in coherent light scattering. The latter resides in cross interference between fluctuational changes in the linear multipole polarizabilities and fluctuational changes in the nonlinear multipole polarizabilities of the molecules. In a first approximation, the contributions derived are purely binary in nature and appear to be highly significant compared with the hitherto calculated pure and cross dipole and multipole contributions to integral light scattering [2-7].

As done earlier for the case of collision-induced far-infrared absorption spectra [8-10], analyses in the multipole approach have appeared recently for the spectra of light scattered by tetrahedral [11-12], axially-symmetric [13], and unlike polar arbitrarily symmetric molecules [14]. However, cross-fluctuation contributions caused by the above mechanism in spectral light scattering have, hitherto, not been considered. 
2. Time-correlation intensity function of isotropic and anisotropic light scattering. - We consider a stochastic medium of volume $V$ containing $N=\sum_{i} N_{i}$ unlike molecules (with $N_{i}$ - the number density of molecules of the $i$-th species). The molecules are assumed to interact, the range of radial correlations being sufficiently short compared with the light wavelength for the phase interference factors to be negligible. The evolution in time of isotropic $(h=0)$ and anisotropic $(h=2)$ light scattering is given by the following time-correlation functions [14] :

$$
C^{(h)}(t)=\sum_{i j}\left\langle\sum_{p=1}^{N_{i}} \sum_{q=1}^{N_{j}} \Pi_{i}^{(h)}\left(\mathbf{r}_{p i}^{0}, \Omega_{p i}^{0}\right)^{*}: \Pi_{j}^{(h)}\left(\mathbf{r}_{q j}^{t}, \Omega_{q j}^{t}\right)\right\rangle
$$

where $\Pi_{i}^{(h)}\left(\mathbf{r}_{p i}^{0}, \Omega_{p i}^{0}\right)$ is the $h$-irreducible part of the second-rank tensor of linear polarizability (dipolar in the case of an electric dipole transition) of a molecule $p$ of species $i$ having, in the medium, the position $\mathbf{r}_{p i}^{0}$ and orientation $\Omega_{p i}^{0}$ at time $t=0$. In equation (1), the symbol $\langle\cdots\rangle$ denotes the appropriate statistical-time averaging.

In a dense medium, collisional effects as well as time- and space-fluctuations of multipolar molecular fields will in general lead to changes in the molecular polarizability tensor [2-6] :

$$
\Pi_{i}\left(\mathbf{r}_{p i}^{t}, \Omega_{p i}^{t}\right)=A_{i}\left(\Omega_{p i}^{t}\right)+\Delta \Pi_{i}^{\mathrm{L}}\left(\mathbf{r}_{p i}^{t}, \Omega_{p i}^{t}\right)+\Delta \Pi_{i}^{\mathrm{NL}}\left(\mathbf{r}_{p i}^{t}, \Omega_{p i}^{t}\right)
$$

where $A_{i}\left(\Omega_{p i}^{t}\right)={ }^{(1)} A_{i}^{(1)}\left(\Omega_{p i}^{t}\right)$ is the second-rank tensor of linear polarizability of an isolated molecule $p$ of species $i$ having the orientation $\Omega_{p i}^{t}$ at time $t$.

In a first approximation, the long-range field-induced change, related with the linear multipolar polarizabilities, is $[1,3,6,14]$ :

$$
\Delta \boldsymbol{\Pi}_{i}^{\mathrm{L}}\left(\mathbf{r}_{p i}^{t}, \Omega_{p i}^{t}\right)=\sum_{k} \sum_{r \neq p}^{N_{k}} \sum_{m=1}^{\infty} \sum_{n=1}^{\infty}(-1)^{n} \frac{2^{m+n} m ! n !}{(2 m) !(2 n) !}{ }^{(1)} \mathbf{A}_{i}^{(m)}\left(\Omega_{p i}^{t}\right)[m]^{(m)} \mathbf{T}_{i k}^{(n)}\left(\mathbf{r}_{p i r k}^{t}\right)[n]^{(n)} \mathbf{A}_{k}^{(1)}\left(\Omega_{r k}^{t}\right),
$$

where the tensor ${ }^{(1)} \mathbf{A}_{i}^{(m)}\left(\Omega_{p i}^{t}\right)$ of rank $1+m$ determines the linear dipolar polarizability due to a $2^{m}$-pole electric transition and the symbol $[m]$ denotes $m$-fold contraction. Whereas the tensor of rank $m+n$

$$
{ }^{(m)} \mathbf{T}_{i k}^{(n)}\left(r_{p i r k}^{t}\right)=\nabla_{p}^{m} \nabla_{r}^{n}\left(r_{p i r k}^{t}\right)^{-1}
$$

defines $2^{m}$-pole $\rightarrow 2^{n}$-pole interaction between molecules pi and $r k$ separated by a distance $r_{p i r k}$.

In equation (2), the additional variation due to the nonlinear polarizabilities, induced by multipolar fields, is defined in a first approximation as $[1,3,6]$ :

$$
\begin{aligned}
\Delta \Pi_{i}^{\mathrm{NL}}\left(\mathbf{r}_{p i}^{t}, \Omega_{p i}^{t}\right)=\sum_{k} \sum_{r \neq p}^{N_{k}} \sum_{m=1}^{\infty} \sum_{n=1}^{\infty}(-1)^{n} \frac{2^{m+n} m ! n !}{(2 m) !(2 n) !} \times \\
\quad \times{ }^{(1)} \mathbf{B}_{i}^{(1+m)}\left(\Omega_{p i}^{t}\right)[m]^{(m)} \mathbf{T}_{i k}^{(n)}\left(\mathbf{r}_{p i r k}^{t}\right)[n] \mathbf{M}_{k}^{(n)}\left(\Omega_{r k}^{t}\right),
\end{aligned}
$$

where the tensor ${ }^{(1)} \mathbf{B}_{i}^{(1+m)}\left(\Omega_{p i}^{t}\right)$ of rank $2+m$ determines the nonlinear (second-order) dipoledipole polarizability due to a $2^{m}$-pole electric transition in a molecule $p$ of species $i . \mathbf{M}_{k}^{(n)}\left(\Omega_{r k}^{t}\right)$ defines the $2^{n}$-pole intrinsic electric moment of molecule $r$ of species $k$.

We refrain from considering here multipolar contributions of higher perturbational approximations (see $[1,3,6])$.

With regard to (2)-(5), the correlation functions (1) can be written in the form of an expansion in powers of the molar fractions $x_{i}(h=0,2)$ :

$$
C^{(h)}(t)=\sum_{i} x_{i} C_{i}^{(h)}(t)+\sum_{i j} x_{i} x_{j} C_{i j}^{(h)}(t)+\sum_{i j k} x_{i} x_{j} x_{k} C_{i j k}^{(h)}(t),
$$


where $C_{i}^{(h)}(t)$ are self-correlation functions describing incoherent scattering by statistically independent molecules possessing intrinsic linear polarizabilities given by the first term of (2). The higher correlation functions $C_{i j}^{(h)}(t), C_{i j k}^{(h)}(t), \ldots$ originate in binary, ternary, etc., molecular correlations, and determine coherent scattering - a measure of the deviation from additivity of the correlation functions given by the first term of (6).

The absolute values of the successive terms of (2) give the pure contributions to the correlation functions (1) whereas the interferential terms give cross-contributions. In this Communication we shall analyse certain novel contributions to the binary correlation function arising by interference between (as the products of) the changes in polarizability (3) and (5). Thus, with regard to (2), these fluctuational cross-contributions to $C_{i j}^{(h)}(t)$ result from the two products $\left\langle\Delta \Pi_{i}^{\mathrm{L}} \Delta \Pi_{j}^{\mathrm{NL}}\right\rangle$ and $\left\langle\Delta \Pi_{i}^{\mathrm{NL}} \Delta \Pi_{j}^{\mathrm{L}}\right\rangle$. When calculating these terms, however, we shall have recourse to the simple model of radially correlated molecules the rotational motions of which are assumed to be mutually independent, meaning that the generalized space-time binary Van Hove distribution distinct function can be approximated as follows :

$$
G_{i j}^{\mathrm{D}}\left(\mathbf{r}_{p i}^{0}, \mathbf{r}_{q j}^{t}, \Omega_{p i}^{0}, \Omega_{q j}^{t}, t\right)=G_{i j}^{\mathrm{D}}\left(\mathbf{r}_{p i}^{0}, \mathbf{r}_{q j}^{t}, t\right) . G_{i}^{\mathrm{S}}\left(\Omega_{p i}^{0}, \Omega_{p i}^{t}, t\right) G_{j}^{\mathrm{S}}\left(\Omega_{q j}^{0}, \Omega_{q j}^{t}, t\right),
$$

where $G_{i j}^{\mathrm{D}}\left(\mathbf{r}_{p i}^{0}, \mathbf{r}_{q j}^{t}, t\right)$ is the usual Van Hove binary radial distribution function, whereas $G_{i}^{\mathbf{S}}\left(\Omega_{p i}^{0}, \Omega_{p i}^{t}, t\right)$ and $G_{j}^{\mathbf{S}}\left(\Omega_{q j}^{0}, \Omega_{q j}^{t}, t\right)$ are the self-distribution functions of the free rotational motions of molecules $p i$ and $q j$.

On insertion of (2)-(5) into (1) and first performing unweighted rotational averaging over all possible molecular orientations in accordance with the approximation (7), we obtain the following binary correlation functions of isotropic and anisotropic light scattering resulting from the term $\left\langle\Delta \Pi_{i}^{\mathrm{L}} \Delta \Pi_{j}^{\mathrm{NL}}\right\rangle$ :

$$
\begin{aligned}
& C_{i j}^{(0)}(t)_{\mathrm{L}, \mathrm{NL}}=-\frac{2}{3} \sum_{m=1}^{\infty} \sum_{n=1}^{\infty} K_{m n} R_{i j}^{(2 m+2 n+4)}(t) \times \\
& \times\left\{\left\langle\left(\mathbf{U}:{ }^{(1)} \mathbf{A}_{i}^{(1+m)}\right)^{*}[m]\left(\mathbf{U}:{ }^{(1)} \mathbf{B}_{i}^{(1+m)}\right)\right\rangle_{\Omega_{i}}\left\langle{ }^{(n)} \mathbf{A}_{j}^{(1)^{*}}[n+1] \mathbf{M}_{j}^{(n+1)}\right\rangle_{\Omega_{j}}+\langle i \leftrightarrow j\rangle+\right. \\
& \left.+\left\langle{ }^{(1)} \mathbf{A}_{i}^{(m)^{*}}[1+m]\left(\mathbf{U}:{ }^{(1)} \mathbf{B}_{i}^{(2+m)}\right)\right\rangle_{\Omega_{i}}\left\langle\left({ }^{(n+1)} \mathbf{A}_{j}^{(1)}: \mathbf{U}\right)^{*}[n] \mathbf{M}_{j}^{(n)}\right\rangle_{\Omega_{j}}+\langle i \leftrightarrow j\rangle\right\} \\
& C_{i j}^{(2)}(t)_{\mathrm{L}, \mathrm{NL}}+C_{i j}^{(0)}(t)_{\mathrm{L}, \mathrm{NL}}=-2 \sum_{m=1}^{\infty} \sum_{n=1}^{\infty} K_{m n} R_{i j}^{(2 m+2 n+4)}(t) \times \\
& \times\left\{\left\langle{ }^{(1)} \mathbf{A}_{i}^{(1+m)^{*}}[2+m]^{(1)} \mathbf{B}_{i}^{(1+m)}\right\rangle_{\Omega_{i}}\left\langle{ }^{(n)} \mathbf{A}_{j}^{(1)^{*}}[n+1] \mathbf{M}_{j}^{(n+1)}\right\rangle_{\Omega_{j}}+\langle i \leftrightarrow j\rangle+\right. \\
& \left.+\left\langle{ }^{(1)} \mathbf{A}_{i}^{(m)^{*}}[1+m]\left({ }^{(1)} \mathbf{B}_{i}^{(2+m)}: \mathbf{U}\right)\right\rangle_{\Omega_{i}}\left\langle\left({ }^{(n+1)} \mathbf{A}_{j}^{(1)}: \mathbf{U}\right)^{*}[n] \mathbf{M}_{j}^{(n)}\right\rangle_{\Omega_{j}}+\langle i \leftrightarrow j\rangle\right\}
\end{aligned}
$$

with the notation :

$$
K_{m n}=\frac{2^{m+n} m !(m+1) ! n !(n+1) !(2 m+2 n+2) !}{(2 m) !(2 m+3) !(2 n) !(2 n+3) !}
$$

and $\mathbf{U}$ - the unit second-rank tensor; and the symbols \langle\rangle$_{\Omega_{i}}$ and \langle\rangle$_{\Omega_{j}}$ denote the rotational averages with the time self-functions $G_{i}^{\mathbf{S}}(t)$ and $G_{j}^{\mathbf{S}}(t)$ involved in equation (7).

In (8) and (9), we have introduced the time radial binary correlation functions :

$$
R_{i j}^{(2 m+2 n+4)}(t)=\rho^{2} \iint\left(r_{p i q j}^{0} r_{p i q j}^{t}\right)^{-(m+n+2)} P_{m+n+1}\left(\frac{\mathbf{r}_{p i q j}^{0} \cdot \mathbf{r}_{p i q j}^{t}}{r_{p i q j}^{0} r_{p i q j}^{t}}\right) G_{i j}^{\mathrm{D}}\left(\mathbf{r}_{p i}^{0}, \mathbf{r}_{q j}^{t}, t\right) \mathrm{d} \mathbf{r}_{p i}^{0} \mathrm{~d} \mathbf{r}_{q j}^{t},
$$

with $P_{m+n+1}$ - the Legendre polynomial of degree $m+n+1$ and $\rho$ - the averaged number density of molecules. 
We obtain directly in analytical form the new contributions $C_{i j}^{(h)}(t)$ originating in the second term $\left\langle\Delta \Pi_{i}^{\mathrm{NL}} \Delta \Pi_{j}^{\mathrm{L}}\right\rangle$ from equations (8) and (9) by an interchange in the indices $i$ and $j$. The total binary contribution to (6) thus amounts to $C_{i j}^{(h)}(t)=C_{i j}^{(h)}(t)_{\mathrm{L}, \mathrm{NL}}+C_{i j}^{(h)}(t)_{\mathrm{NL}, \mathrm{L}}$.

The cognitional significance of the new contributions (8)-(10) resides i.a. in the fact that they are negative, and can undergo a change in sign only if the tensor of one of the multipolar polarizabilities or intrinsic multipole moment is negative, as can be the case for certain molecular structures.

3. Applications to some simple molecular models. - The correlation functions (8) and (9) contain all cross-fluctuation contributions arising by interference of linear and nonlinear multipole polarizabilities. We shall now apply them to some simple models of molecules in successive multipole approximations. For the sake of simplicity, we shall restrict our considerations to real values of the tensors of multipole polarizabilities (written in irreducible representation) and omit the explicit time-rotational dependences in \langle\rangle$_{\Omega_{i}}$ and \langle\rangle$_{\Omega_{j}}$.

3.1 QUADRUPOle APPROXIMATION. - The lowest-order contributions of (8) and (9) arise for $m=n=1$, when we have for axially symmetric molecules having an intrinsic dipole moment $M_{3}$ :

$$
\begin{aligned}
& C_{i j}^{(0)}(t)=-\frac{15}{2}\left(A_{2}^{(i)} B_{14}^{(i)} A_{1}^{(j)} M_{3}^{(j)}+A_{2}^{(j)} B_{14}^{(j)} A_{1}^{(i)} M_{3}^{(i)}\right) R_{i j}^{(8)}(t), \\
& C_{i j}^{(2)}(t)=-\frac{15}{2}\left\{\left(A_{0}^{(i)} B_{04}^{(i)}+A_{2}^{(i)} B_{24}^{(i)}\right) A_{1}^{(j)} M_{3}^{(j)}+\left(A_{0}^{(j)} B_{04}^{(j)}+A_{2}^{(j)} B_{24}^{(j)}\right) A_{1}^{(i)} M_{3}^{(i)}\right\} R_{i j}^{(8)}(t),
\end{aligned}
$$

where we have two irreducible parameters $A_{0}=\left(A_{33}+2 A_{11}\right) / 3$ and $A_{2}=2\left(A_{33}-A_{11}\right) / 3$ of the linear dipole-dipole polarizability ${ }^{(1)} \mathrm{A}^{(1)} \rightarrow A_{\alpha \beta}$, one parameter $A_{1}=2\left(A_{3: 33}+2 A_{1: 13}\right) / 5$ of the linear dipole-quadrupole polarizability tensor ${ }^{(1)} \mathbf{A}^{(2)} \rightarrow A_{\alpha: \beta \gamma}$ and three parameters

$$
B_{04}=2\left(B_{33: 33}+4 B_{13: 13}+B_{11: 33}+4 B_{11: 11}\right) / 15, \quad B_{14}=\left(B_{33: 33}+2 B_{11: 33}\right) / 15,
$$

$B_{24}=\left(B_{33: 33}+2 B_{13: 13}-3 B_{11: 33}-4 B_{11: 11}\right) / 15$ of the nonlinear dipole-quadrupole polarizability tensor ${ }^{(1)} B^{(1+2)} \rightarrow B_{\alpha \beta: \gamma \delta}$.

In the same approximation we have by (8) and (9) for axially symmetric molecules with intrinsic quadrupole moment $M_{33}$ :

$$
\begin{aligned}
C_{i j}^{(0)}(t)= & -\frac{5}{6}\left\{A_{1}^{(i)} B_{1}^{(i)} A_{2}^{(j)} M_{33}^{(j)}+A_{1}^{(j)} B_{1}^{(j)} A_{2}^{(i)} M_{33}^{(i)}\right\} R_{i j}^{(8)}(t) \\
C_{i j}^{(2)}(t)_{\mathrm{L}, \mathrm{NL}}=-\frac{1}{6}\left\{\left(A_{1}^{(i)} B_{1}^{(i)}+9 A_{3}^{(i)} B_{3}^{(i)}\right) A_{2}^{(j)} M_{33}^{(j)}+\left(A_{1}^{(j)} B_{1}^{(j)}\right.\right. & \left.+9 A_{3}^{(j)} B_{3}^{(j)}\right) \\
\times & \left.\times A_{2}^{(i)} M_{33}^{(i)}\right\} R_{i j}^{(8)}(t),
\end{aligned}
$$

with the following notation of the irreducible molecular parameters :

$$
A_{3}=\left(3 A_{3: 33}-4 A_{1: 13}\right) / 5, \quad B_{1}=3\left(B_{333}+2 B_{113}\right) / 5, \quad B_{3}=2\left(B_{333}-3 B_{113}\right) / 5 .
$$

We now shall consider the situation arising if the molecule of species (component) $i$ are tetrahedral and those of species $j$ are axially-symmetric. In this case the isotropic scattering correlation function (8) vanishes whereas that of anisotropic scattering (9) takes the simple form

$$
C_{i j}^{(2)}(t)_{\mathrm{L}, \mathrm{NL}}=-\frac{18}{5} A_{1: 23}^{(i)} B_{123}^{(i)} A_{2}^{(j)} M_{33}^{(j)} R_{i j}^{(8)}(t) .
$$


3.2 Octupole approximation. - With $m=1$ and $n=2$, equations (8) and (9) lead to expressions similar to (11a) and (11b) in which, in place of $A_{1} M_{3} R_{i j}^{(8)}(t)$, we have a term $4\left(A_{3: 333}+2 A_{1: 133}\right) / 5 M_{33} R_{i j}^{(10)}(t)$ involving the dipole-octupole polarizability ${ }^{(1)} \mathrm{A}^{(3)} \rightarrow A_{\alpha: \beta \gamma \delta}$. For the case of tetrahedral molecules $i$ with octupole moment $\boldsymbol{M}_{123}^{(i)}$ and axially-symmetric molecules $j$ with octupole moment $M_{333}^{(j)}$, equations (8) and (9) yield :

$$
\begin{aligned}
& C_{i j}^{(0)}(t)_{\mathrm{L}, \mathrm{NL}}=-\frac{40}{9} A_{1: 23}^{(i)} M_{123}^{(i)} A_{1}^{(j)} B_{1}^{(j)} R_{i j}^{(10)}(t), \\
& C_{i j}^{(2)}(t)_{\mathrm{L}, \mathrm{NL}}=-\frac{8}{9} A_{1: 23}^{(i)}\left\{9 B_{123}^{(i)} A_{3}^{(j)} M_{333}^{(j)}+M_{123}^{(i)}\left(A_{1}^{(j)} B_{1}^{(j)}+9 A_{3}^{(j)} B_{3}^{(j)}\right)\right\} R_{i j}^{(10)}(t) .
\end{aligned}
$$

In the same approximation, albeit for the case when the molecules of both components are tetrahedral, the isotropic scattering function vanishes and the anisotropic scattering function becomes :

$$
C_{i j}^{(2)}(t)_{\mathrm{L}, \mathrm{NL}}=-\frac{96}{5} A_{123}^{(i)} A_{123}^{(j)}\left(B_{123}^{(i)} M_{123}^{(j)}+B_{123}^{(j)} M_{123}^{(i)}\right) R_{i j}^{(10)}(t) .
$$

Putting $m=2$ and $n=1$ in (8) and (9) and assuming the molecules $i$ as tetra- or octahedral and those of species $j$ as axially-symmetric, we obtain $C_{i j}^{(0)}(t)_{\mathrm{L}, \mathrm{NL}}=0$ and

$$
C_{i j}^{(2)}(t)_{\mathrm{L}, \mathrm{NL}}=-6 A_{3: 333}^{(i)} B_{4}^{(i)} A_{2}^{(j)} M_{33}^{(j)} R_{i j}^{(10)}(t),
$$

where $A_{3: 333}^{(i)}$ is the only independent tensor component of the linear dipole-octupole polarizability $A_{\alpha: \beta \gamma \delta}^{(i)}$ whereas $B_{4}^{(i)}=\left(3 B_{33: 33}^{(i)}-4 B_{12: 13}^{(i)}\right) / 5$ is the irreducible anisotropic parameter of the nonlinear dipole-quadrupole polarizability tensor $B_{\alpha \beta: \gamma \delta}^{(i)}$ for the point group symmetries $\mathrm{T}, \mathrm{O}$, and $\mathrm{O}_{\mathrm{h}}[13]$.

Similarly, by (8) and (9), we find for $m=n=2$ in the case of a mixture of octahedral molecules of species $i$ and tetrahedral molecules of species $j, C_{i j}^{(0)}(t)_{\mathrm{L}, \mathrm{NL}}=0$ and

$$
C_{i j}^{(2)}(t)_{\mathrm{L}, \mathrm{NL}}=-\frac{360}{7} A_{3: 333}^{(i)} B_{4}^{(i)} A_{1: 23}^{(j)} M_{123}^{(j)} R_{i j}^{(12)}(t) .
$$

3. 3 HeXADECAPOLE APPROXimATION. - In the approximation of $m=1, n=3$ for octahedral molecules $i$ with a hexadecapole moment $M_{3333}^{(i)}$ and tetrahedral molecules $j$, equations (8) and (9) lead to :

$$
C_{i j}^{(2)}(t)_{\mathrm{L}, \mathrm{NL}}=-24 A_{3: 333}^{(i)} M_{3333}^{(i)} A_{1: 23}^{(j)} B_{123}^{(j)} R_{i j}^{(12)}(t) .
$$

Finally, for $m=2$ and $n=3$ in the case when the molecules of both components are octahedral, we obtain :

$$
C_{i j}^{(2)}(t)_{\mathrm{L}, \mathrm{NL}}=-\frac{660}{7} A_{3: 333}^{(i)} A_{3: 333}^{(j)}\left(B_{4}^{(i)} M_{3333}^{(j)}+B_{4}^{(j)} M_{3333}^{(i)}\right) R_{i j}^{(14)}(t) .
$$

4. Discussion and conclusions. - The special formulae (11)-(19) derived by us are well adapted to numerical evaluations since the irreducible parameters of the multipole polarizabilities and the intrinsic moments are available from the literature $[9,10,15,16]$. The evaluations confirm the following obvious relationship between the two-body contributions

$$
C_{i j}^{(h)}\left(t_{\mathrm{L}^{2}}>C_{i j}^{(h)}(t)_{\mathrm{L}, \mathrm{NL}}+C_{i j}^{(h)}(t)_{\mathrm{NL}, \mathrm{L}}>C_{i j}^{(h)}(t)_{(\mathrm{NL})^{2}} .\right.
$$


E.g., for $\mathrm{CO}_{2}$, the ratio of the relative values of the contributions is $9: 3: 1$ for isotropic scattering. However, it should be stressed that the role of the binary cross-contributions analysed in this Communication can be strongly enhanced owing to the circumstance that the values of the binary pure contributions of the types $(\Delta \mathrm{L})^{2}$ and $(\Delta \mathrm{NL})^{2}$ can undergo a considerable reduction by negative ternary contributions [6].

The general conclusion to be drawn from the present analysis as well as our earlier calculations $[6,14]$ is this : when analysing the role of multipolar molecular interactions in coherent light scattering, all possible contributions have to be taken into account. Depending on the case under consideration, they can cancel out or enhance mutually.

\section{References}

[1] Kielich, S., Mol. Phys. 9 (1965) 549; Acta Phys. Pol. 27 (1965) 305; 28 (1965) 95.

[2] Kielich, S., Acta Phys. Pol. 19 (1960) 711 ; J. Physique 29 (1968) 619; Chem. Phys. Lett. 10 (1971) $516 ; 19$ (1973) 609.

[3] Berrue, J., Chave, A., Dumon, B. and Thibeau, M., J. Physique 36 (1975) 1079.

[4] Ladanyi, B. M. and Keyes, T., Mol. Phys. 33 (1977) $1063 ; 1247$.

[5] Pasmanter, R. A., Samson, R. and Ben-Reuven, Phys. Rev. 14A (1976) 1238.

[6] Kielich, S., Opt. Commun. 34 (1980) 367.

[7] Oksengorn, B., C. R. Hebd. Séan. Acad. Sci. Paris B 282 (1976) 223.

[8] Gray, C. G., J. Phys. B 4 (1971) 1661.

[9] Isnard, P., Robert, D. and Galatry, L., Mol. Phys. 39 (1980) 501.

[10] Birnbaum, G. and Sutter, H., Mol. Phys. 42 (1981) 21.

[11] Posch, H. A., Mol. Phys. 37 (1979) 1059; 40 (1980) 1137.

[12] Buckingham, A. D. and Tabisz, G. C., Opt. Lett. 1 (1977) 220.

[13] Bancewicz, T., Acta Phys. Pol. A 56 (1979) 431 ;

Frenkel, D. and McTague, J. P., J. Chem. Phys. 72 (1980) 2801.

[14] Kielich, S., Bancewicz, T. and Woźniak, S., Can. J. Phys. 59 (1981) 1600.

[15] Kielich, S., Molecular Nonlinear Optics (Izd. Nauka, Moscow) 1981.

[16] Amos, R. D., Mol. Phys. 38 (1979) 33 ; 39 (1980) 1. 